\title{
Differences in the "egumi" taste of moso-bamboo shoots: research using chemical analysis and two types of taste sensors
}

Yuka Furusawa ${ }^{1,2^{*}}$, Hitoshi Nakamura ${ }^{2}$ and Tatsuya Ashitani ${ }^{1,3}$

\begin{abstract}
We conducted our research with the aim of determining whether the "egumi" taste of moso-bamboo shoots differs depending on the production area and how the "egumi" taste of bamboo shoots from Yamagata Prefecture, which is near the northern limit of cultivation, differs. The investigation was conducted using conventional chemical analysis methods to quantitatively evaluate the presence and content of homogentisic acid and oxalic acid in bamboo shoots from different regions. In addition, qualitative evaluation, for which chemical analysis alone is insufficient, was conducted using taste sensors, which have rarely been used. As a result, chemical analysis showed that homogentisic acid was not identified among the two substances considered to be major components of egumi taste, while oxalic acid was identified in all samples. Therefore, it is highly likely that oxalic acid is responsible for the "egumi" taste in this study. In addition, qualitative analysis using taste sensors revealed that there is a difference in "egumi" taste depending on the origin of the bamboo shoot. It was suggested that bamboo shoots from Yamagata Prefecture, near the northern limit of cultivation, had a stronger "egumi" taste than those from Kyoto and Fukuoka Prefectures.
\end{abstract}

Keywords: Moso-bamboo, Bamboo shoots, Place of origin, "Egumi" taste

\section{Introduction}

Moso-bamboo (Phyllostachys edulis) is the largest species of bamboo in Japan and was introduced in the seventeenth century for food, having edible young shoots (bamboo shoots), and for materials [1]. In recent years, the number of bamboo forests that are not being used properly has been increasing due to a decrease in the use of bamboo materials, and in order to solve these problems, new applications are being developed, such as the production of rayon, the development of technology for the production of cellulose nanofibers, a new material made from bamboo, and products using the extracted liquid [2]. On the other hand, bamboo shoot production is a valuable income sources because it provides an annual

\footnotetext{
*Correspondence: furusaway@pref.yamagata.jp

${ }^{1}$ The United Graduate School of Agricultural Sciences, Iwate University,

3-18 Ueda, Morioka, Iwate 020-0066, Japan

Full list of author information is available at the end of the article
}

income during the long period of timber production [3], and bamboo shoots are produced widely throughout Japan. The production of bamboo shoots in Japan is done mainly south of the Kansai region, which accounts for $89.5 \%$ of the national total [4], and the Tohoku region is only $1.4 \%$, owing to its proximity to the northern cultivation limit. In 2019, the production volume in Yamagata Prefecture was the largest in the Tohoku region, 193.7 tons [4].

In Yamagata Prefecture, the supply of locally collected bamboo shoots has not kept up with demand. Currently, these locally collected bamboo shoots are mainly consumed within the prefecture [5], but there is a movement to brand bamboo shoots in each production area, and producers are highly interested in this "value added" branding. There are several factors to be considered when differentiating bamboo shoots from other regions, such as shipping time, size, quality, and functional ingredients. The taste is also an important unique characteristic. 
Bamboo shoot growers sensibly feel that the taste of bamboo shoots, especially "egumi" taste, varies depending on the production area where they are grown, and there is a strong interest in the difference between regions within the same prefecture and the difference between bamboo shoots produced in snow-covered areas near the northern limit of cultivation and those produced in other prefectures.

To date, studies on the taste of moso-bamboo have been based mostly on chemical analyses of related substances [6-10], and the major components responsible for the "egumi" taste have been reported to be homogentisic acid and oxalic acid [6, 11]. However, while chemical analyses may show quantitative differences in taste-related substances, they are insufficient to describe qualitative differences in detail. There is a huge variety of taste-related substances, and their interactions cannot be ascertained through chemical analyses [12-14]. Therefore, in general, the taste of food is judged by sensory testing, but this testing is also problematic. It requires qualified inspectors, who are biased by their own opinions and physical conditions. In addition, there are differences in what constitutes an "appealing" taste to consumers from various cultures and traditions [12, 15, 16]. For this reason, in recent years, objective taste sensors have been used to complement chemical analyses and sensory evaluations in quality differentiation and new product development [13, 14, 16-18]. In the field of forestry, the taste of nameko mushrooms was quantified to objectively evaluate their taste [19].

In this study, we focused on the taste of bamboo shoots, which growers are interested in. In particular, we aimed to clarify whether the "egumi" taste differs depending on the production area, and how the "egumi" taste of bamboo shoots produced in Yamagata Prefecture, which is close to the northern limit of cultivation, differs from the taste of bamboo shoots produced in other regions. To clarify this point, we quantitatively evaluated the presence and content of homogentisic acid and oxalic acid in bamboo shoots grown in and outside of the prefecture using conventional chemical analysis. In addition, qualitative evaluation, which is insufficient for chemical analysis, was conducted using taste sensors, which have rarely been used in the past.

\section{Materials and methods}

\section{Material collection and sample preparation}

Bamboo shoots were collected from eight locations (Fig. 1), six of which were located in Yamagata Prefecture. Four were collected from the Shonai region, which is the main production area in the prefecture, one in Yuza Town, one in Mikawa Town, and two in Tsuruoka City (Sanze and Haguro), while the other two were collected from the inland region, one in Yamanobe Town and one in Asahi Town. To compare the results with those of other prefectures, one site in Kyoto Prefecture and one site in Fukuoka Prefecture were selected from the top production regions, which are south of western Japan [4].

Three bamboo shoots were collected from each site. Because the developmental time for bamboo shoots varies from region to region, the collection period was approximately 10 days after the first bamboo shoot was confirmed at each site, which ranged from early to late May in 2017. The size of the bamboo shoots collected was standardized to the Shonai area's standard M (600$1000 \mathrm{~g}$ ) for fresh food shipments [5]. The bamboo shoots were collected from the two locations outside of the prefecture from early to mid-April in 2018, and three bamboo shoots that met the same raw food shipping standard M (600-1000 g) used for those collected within the prefecture were selected.

Fresh edible bamboo shoots collected in the prefecture are distributed mainly through in-prefecture market shipments and direct sales, with the shortest time from collection to sale being approximately one day, and they are rarely kept refrigerated during market management or sales [5]. Therefore, assuming similar distribution conditions, bamboo shoots were transported to the Yamagata Prefecture Forestry Research and Training Center within $3 \mathrm{~h}$ of collection, stored in a well-ventilated area out of direct sunlight until $24 \mathrm{~h}$ after collection, and then fixed by freezing. Specimens collected in Kyoto and Fukuoka prefectures were also transported and stored under conditions similar to those in Yamagata Prefecture, and they were transported by refrigerated vehicle to the Yamagata Prefecture Forest Research and Training Center within 2 days. Upon arrival, the specimens were immediately frozen and fixed.

All the frozen bamboo shoots were freeze-dried for approximately 3 days and used as samples. The freezedrying does not greatly alter the processing methods, and the component levels before drying are mostly maintained [20-22]. Because there is no change in flavor that would cause the food to lose its original characteristics, there is no effect on sensory testing or taste sensor and chemical analyses [20].

Analysis of the "egumi" taste of bamboo shoots by region.

\section{Chemical analysis}

Homogentisic acid and oxalic acid, which are considered to be the main components of "egumi" taste, were analyzed in one sample from Shonai area in Yamagata Prefecture (Yuza Town), one sample from inland area in Yamagata Prefecture (Asahi Town), and one sample from outside the prefecture (Fukuoka Prefecture). 


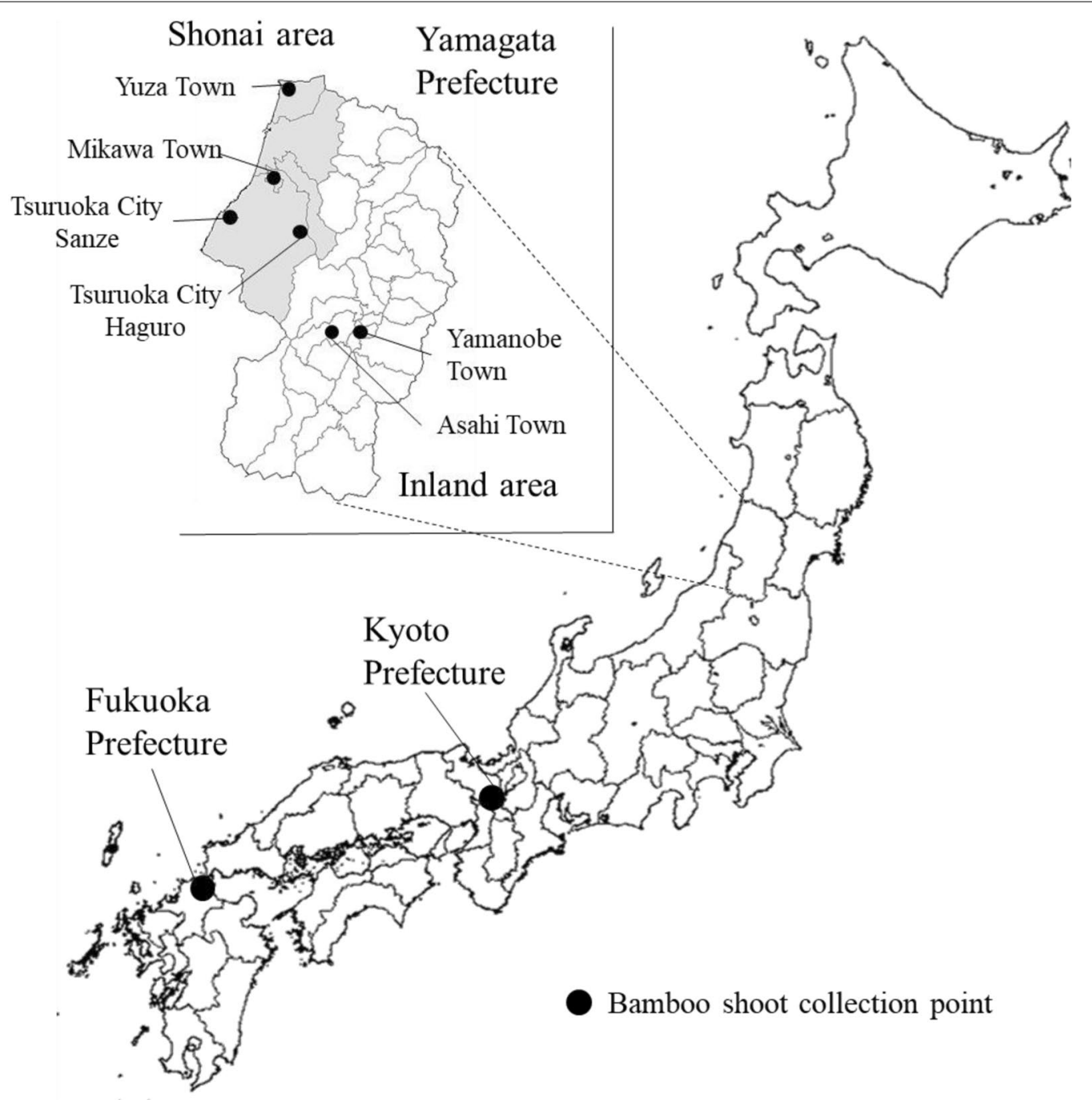

Fig. 1 Bamboo shoot collection sites. Filled circle indicates the locations of the bamboo shoot collection sites. In Yamagata Prefecture, the shaded area indicates the Shonai region, which is the main bamboo shoot production area, and the other area is the inland region

Homogentisic acid was analyzed using gas chromatograph-mass spectrometer (GC-MS). Each of the freezedried samples was degreased, and they were extracted three times by immersion in methanol to recover the supernatant. After concentrating the recovered supernatant, it was transformed into an extract in a constant volume of methanol. The solvent was removed under vacuum conditions to obtain a methanol extract. The methanol extract was analyzed by GC-MS after trimethylsilyl derivatization by TMSI-H [hexamethyldisilazane and trimethylchlorosilane in pyridine $(2: 1: 10, \mathrm{v} / \mathrm{v})]$ (GL Science Inc., Tokyo, Japan) and N,O-bis(trimethylsilyl) acetamide (GL Science Inc., Tokyo, Japan). The GC-MS data were collected with a Shimadzu QP-2010 Ultra-high performance gas chromatograph-mass spectrometer
(Shimadzu, Kyoto, Japan) under the following conditions: SH-Rtx-5ms capillary column $[30 \mathrm{~m} \times 0.25 \mathrm{~mm}$ inner diameter; 0.25- $\mu \mathrm{m}$ film thickness (Shimadzu, Kyoto, Japan)], column temperature ranging from $100{ }^{\circ} \mathrm{C}(1 \mathrm{~min})$ to $320^{\circ} \mathrm{C}(5 \mathrm{~min})$ at $5{ }^{\circ} \mathrm{C} / \mathrm{min}$, injection temperature of $250{ }^{\circ} \mathrm{C}$, interface temperature of $320{ }^{\circ} \mathrm{C}$, acquisition mass range of 50-800 atomic mass units, and helium as the carrier gas.

The concentration of oxalic acid was measured using an E-kit oxalic acid manufactured by J.K. International Co. Measurements were performed on the three selected samples as in GC-MS. $20 \mathrm{~g}$ (fresh weight) of each freezedried sample was weighed, and distilled water was added to form a 20-fold dilution. The treatment time for the test was $6 \mathrm{~h}$, and the samples were refrigerated to avoid 
deterioration owing to temperature. Afterwards, the samples were thoroughly agitated with a mixer and centrifuged at $3000 \mathrm{rpm}$ for $5 \mathrm{~min}$, and the collected supernatants were then used. The supernatants were kept frozen until just before the analyses. The treatment method, spectrophotometry conditions, and formula for calculating the oxalic acid concentration were described in the manual provided with the kit.

\section{Taste analysis}

The samples used were tenfold diluted solutions prepared by the same method as in oxalic acid concentration measurement test.

Two types of taste sensors, $\alpha$ ASTREE (Alpha M.O.S. Japan K.K., Tokyo, Japan) and TS-5000Z (Intelligent Sensor Technology, Inc., Kanagawa, Japan), were used for the analysis.

aASTREE classifies flavors by combining seven types of sensors that exhibit different response spectra to create a unique pattern for each measurement sample [23]. Each sensor consists of a chemical field effect transistor (CHEMFET) base coated with a different copolymer as a sensitive film [23, 24]. In general, most CHEMFET sensors used in industry and research are designed with membranes that respond only to certain ionic species, but the sensors used in $\alpha$ ASTREE have been developed with sensitive materials to induce interactions (such as hydrogen bonds and van der Waals interactions) with a wide range of dissolved taste components, and are sensitive to many ionic and neutral species in complex taste solutions [23, 24]. The potential difference between the CHEMFET sensor and the reference electrode $(\mathrm{Ag} /$ $\mathrm{AgCl}$ ) changes when the sensing membrane comes into contact with the taste component in the sample solution, and is detected as a signal [23, 24]. In $\alpha$ ASTREE, three tastes (sour, salty, and umami) are analyzed directly from the corresponding sensor output, while tastes with other attributes (astringent, bitter, pungent, etc.) need to be defined for each analysis. Analyses using the standard addition method are recommended [23]. In the standard addition method, influential raw materials, and reference materials that are considered to influence the taste to be evaluated, are selected and at least two concentrations are added. In this study, oxalic acid, which was confirmed to be contained in the chemical analysis described above, was used as the target of the standard addition method. Three concentrations of oxalic acid solutions ( $1 \mathrm{mM}, 10 \mathrm{mM}$, and $100 \mathrm{mM}$ ) were used for taste sensor analysis, and principal component analysis was performed along with the analysis results of the samples. In the taste sensor analysis, each sample was measured six times, the average of the two measurements in the second half, when the data was judged to be stable, was used for the principal component analysis. All the principal component analyses were performed using Bell Curve for Excel version 3.21 (Social Survey Research Information Co., Ltd.).

The TS-5000Z employs an artificial lipid membrane taste sensor that mimics the taste recognition mechanism of living organisms [14]. The membrane potential change generated by electrostatic or hydrophobic interactions with the taste substance is detected by the computer as the sensor output, and the potential difference between each sample [25]. The output from each sensor is converted into the corresponding specific "taste item" [25]. The TS-5000Z uses the CPA measurement method, which detects two types of information from a single sensor [25]. Specifically, the potential of the reference solution is set to zero, and the potential difference between the reference solution and the sample solution is measured as a pre-taste [25]. After that, the sensor is lightly cleaned, and the potential difference when the reference solution is measured again is measured as the aftertaste [25].

However, TS-5000Z does not have a sensor that directly measures "egumi" taste, which is not a basic taste. In addition, there is no clear definition of "egumi" taste; it is reported to be a complex mixture of bitter and astringent tastes [26], or a mixture of bitter and astringent tastes with an itchy sensation [27]. For this reason, the measurements of bitterness (first taste), general bitterness (aftertaste), astringent stimulation (first taste), and astringent taste (aftertaste) were included in the analysis in this study. Measurements were made using the three selected samples as in chemical analysis.

\section{Results}

\section{Chemical analysis}

The chromatographs of each sample from Yuza Town (Shonai area in the prefecture), Asahi Town (inland area in the prefecture), and Fukuoka Prefecture (outside the prefecture), as well as the comparison sample, homogentisic acid alone are shown in Fig. 2. Mainly monosaccharides and disaccharides were detected, and homogentisic acid was not detected in any of the three samples. However, tyrosine, the precursor of homogentisic acid, was detected in all the samples (Fig. 2).

The oxalic acid concentrations in samples from Yuza Town (Shonai area within the prefecture), Asahi Town (inland area within the prefecture), and Fukuoka Prefecture (outside the prefecture) were measured using an oxalic acid E-kit. As a result, oxalic acid was detected in all samples (Fig. 3). The oxalic acid concentrations of the samples from Asahi Town, Yuza Town, and Fukuoka Prefecture, were $100.60 \mathrm{mg} / \mathrm{L}, 84.85 \mathrm{mg} / \mathrm{L}$, and $74.71 \mathrm{mg} / \mathrm{L}$, in descending order of concentration (Fig. 3). 


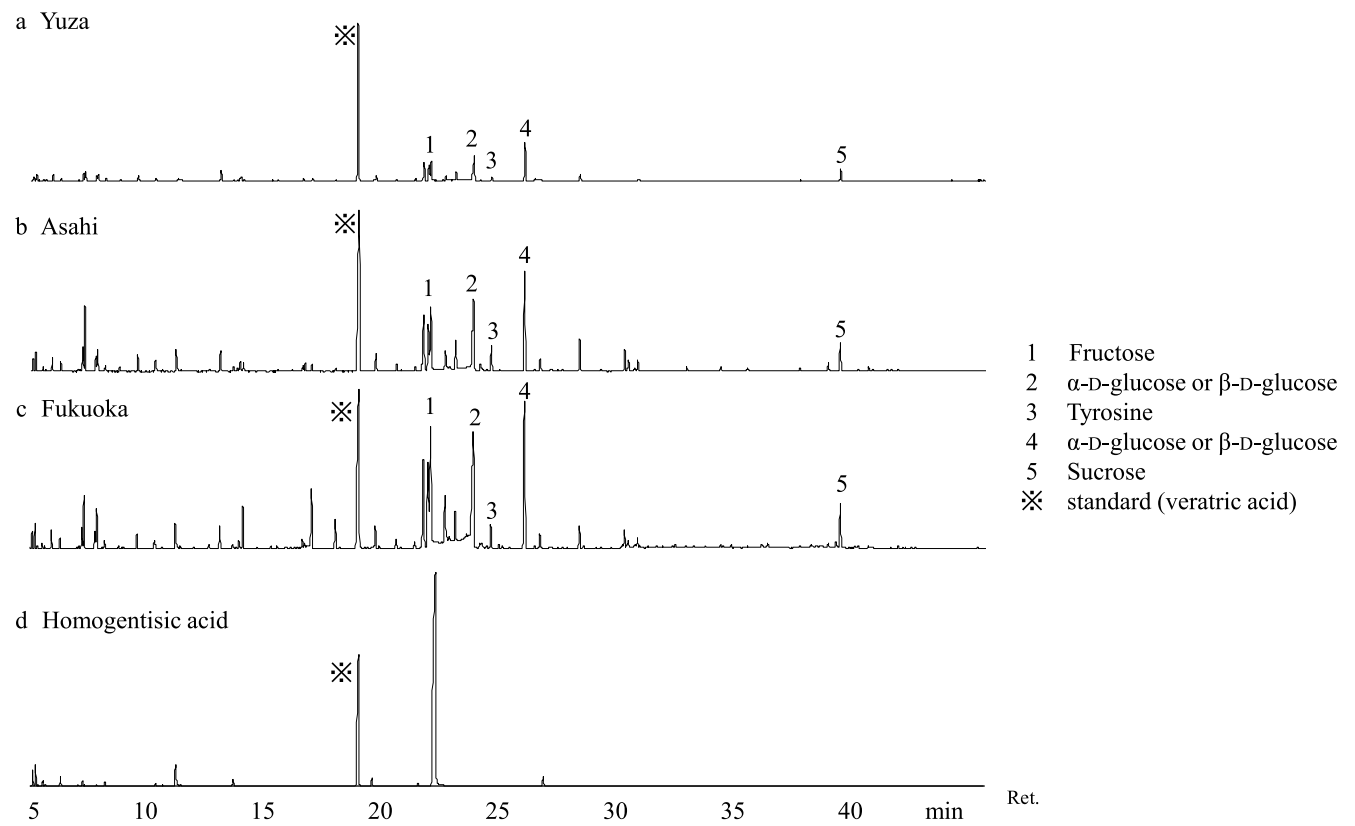

Fig. 2 Chromatogram of the three selected samples and homogentisic acid. Chromatogram of the methanol extracts of the three selected samples and of homogentisic acid for comparison. The substance corresponding to the number assigned to each peak is indicated in the legend. No peaks identical to the homogentisic acid peak were detected in the samples

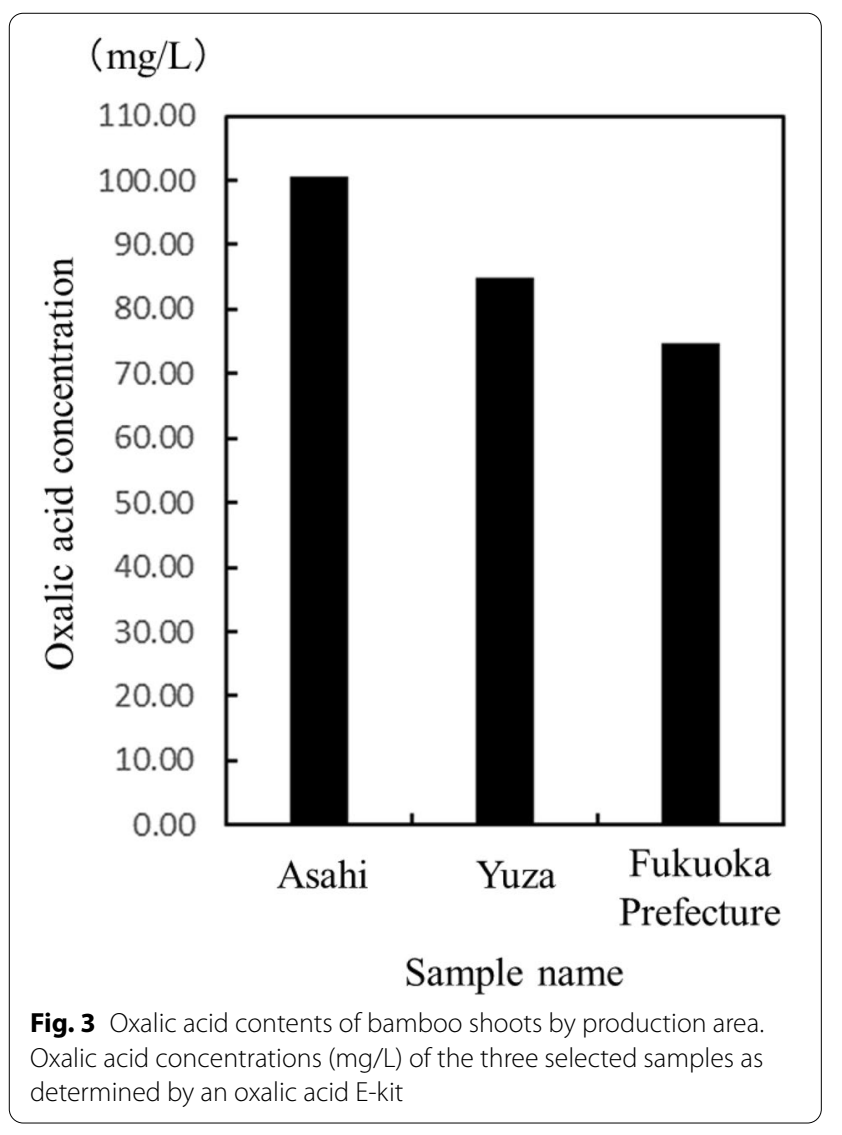

\section{Taste analysis}

At first, the bamboo shoots collected from eight different locations and oxalic acid solutions of three concentrations were measured using $\alpha$ ASTREE, and the results of the principal component analysis are shown in Fig. 4. The contribution of the first principal component was $95.22 \%$ and that of the second principal component was 3.85\%, indicating that the first and second principal components explained approximately $99 \%$ of the results. The differences in taste by region were largely divided between bamboo shoots produced in Yamagata Prefecture and those produced outside the prefecture (Fig. 4). Bamboo shoots produced in Yamagata Prefecture were plotted in almost the same locations regardless of whether they were produced in the Shonai or inland areas, and there was no significant difference in taste (Fig. 4). The three concentrations of oxalic acid solutions were plotted in the direction of increasing first and second principal components as the concentration increased, indicating that the samples from within the prefecture, which were plotted in the upper right direction of the figure, had a stronger taste of oxalic acid (Fig. 4). This result showed the same trend as the results of oxalic acid concentration measurement by chemical analysis (Figs. 3,4 ).

Next, the analysis results using the TS-5000Z are shown in Fig. 5. When the values of bitterness (first taste) and general bitterness (aftertaste) of the sample from 


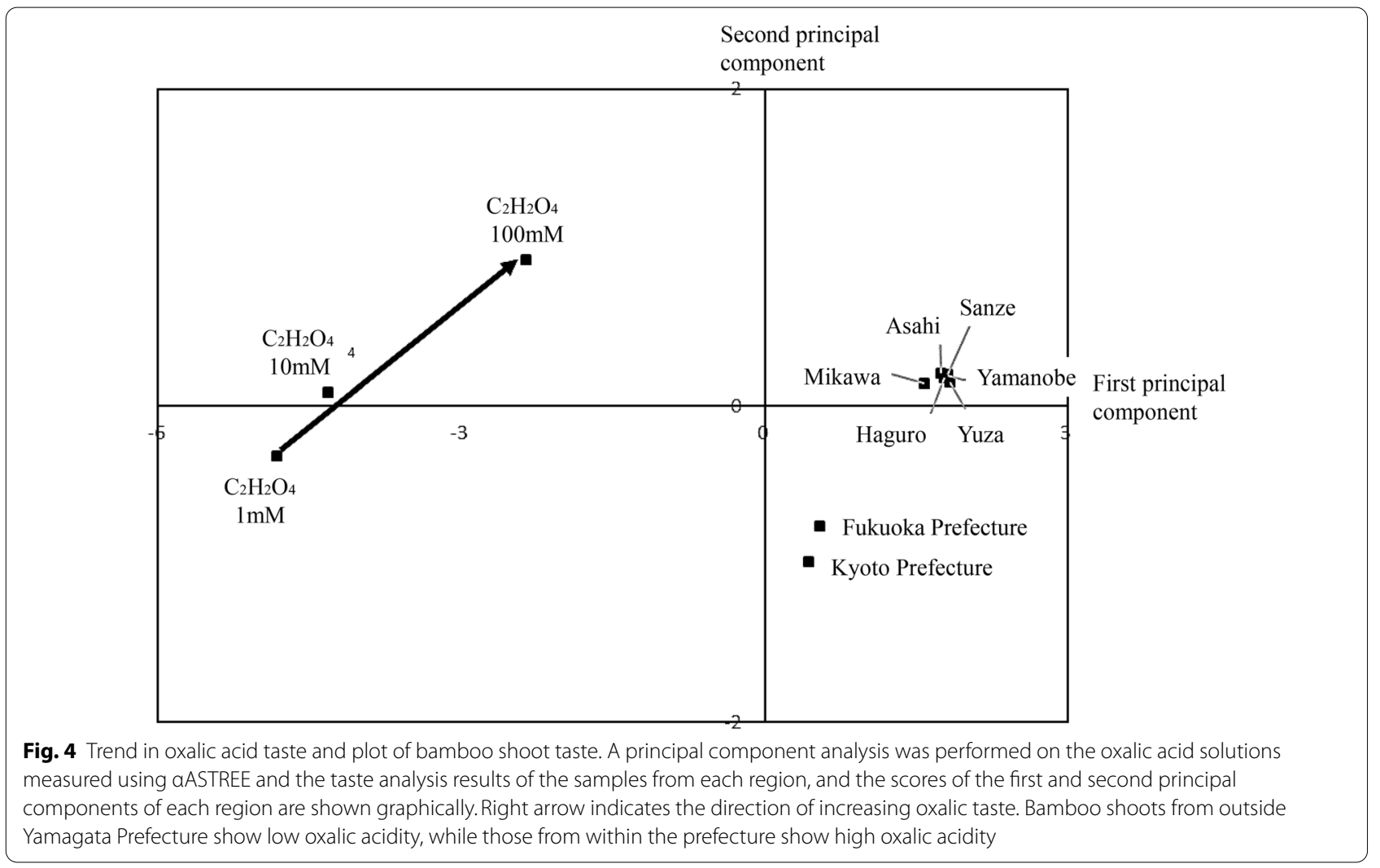

Yuza Town were set at 0.00 , the values of the sample from Asahi Town were 0.16 and 0.25 , respectively (Fig. 5). However, for samples collected in Fukuoka Prefecture, the bitterness (first taste) was -0.48 and general bitterness (aftertaste) was -0.06 , indicating that the contributions of both first taste and aftertaste were small (Fig. 5). This trend was similar to that found using chemical analysis and $\alpha$ ASTREE (Figs. 3, 4, 5). For the astringent stimulation (first taste) and astringent taste (aftertaste), when the value of the sample from Yuza Town was set as 0.00 , the values of the sample from Asahi Town were -0.50 and 0.06 , respectively, indicating that the contribution of the first taste was small and that of the aftertaste was large (Fig. 5). For the sample from within Fukuoka Prefecture, the astringent stimulus (first taste) was 0.18 and the astringent taste (aftertaste) was 0.15. Thus, the contributions of both the first taste and aftertaste were large (Fig. 5). The astringent stimulus (first taste) and astringent (aftertaste) tended to differ from the results of chemical analysis and the trends in oxalic acid concentrations determined using $\alpha$ ASTREE (Figs. 3, 4, 5).

\section{Discussion}

The GC-MS analysis did not detect homogentisic acid, which is considered to be a major source of "egumi" taste (Fig. 2). Homogentisic acid is produced by the deamination of tyrosine to form para-hydroxyphenylpyruvate, which is catalyzed by para-hydroxyphenylpyruvate hydroxylase $[8,28]$. In the present study, only the precursor was detected, suggesting that synthesis may not have advanced. Homogentisic acid levels increase as shoot size decreases, and the acid is most abundant in SS-sized shoots [9]. The samples used in this study were M-size (600-1000 g) bamboo shoots, which are relatively large. Therefore, it is possible that although synthesis had occurred, the amount of homogentisic acid produced was very small, below the detection level.

On the other hand, oxalic acid, another substance that is considered to be responsible for the "egumi" taste, was detected in all samples (Fig. 3). Based on the results of these chemical analyses, it is highly likely that oxalic acid is responsible for the "egumi" taste in this study.

In addition, the taste sensor $\alpha$ ASTREE analysis showed that the taste of bamboo shoots from eight locations was divided into two groups: those from within the prefecture and those from outside the prefecture, with those from within the prefecture having a stronger oxalic acid taste. This result was similar to the results of oxalic acid concentration measurement (Figs. 3, 4). Furthermore, the results of bitterness (first taste) and general bitterness (aftertaste) by the taste sensor TS-5000Z showed the same tendency as the results of oxalic acid concentration 


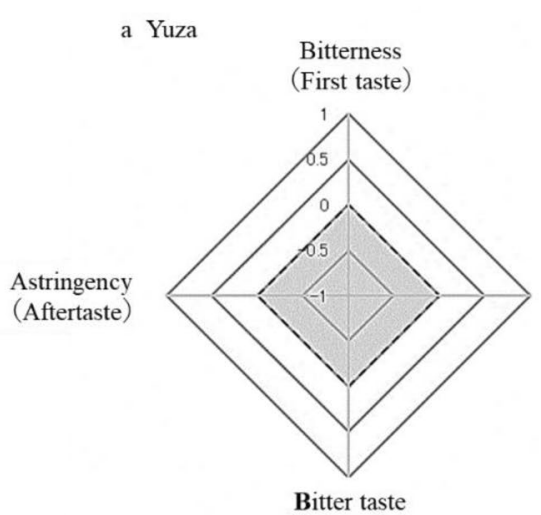

(Aftertaste)

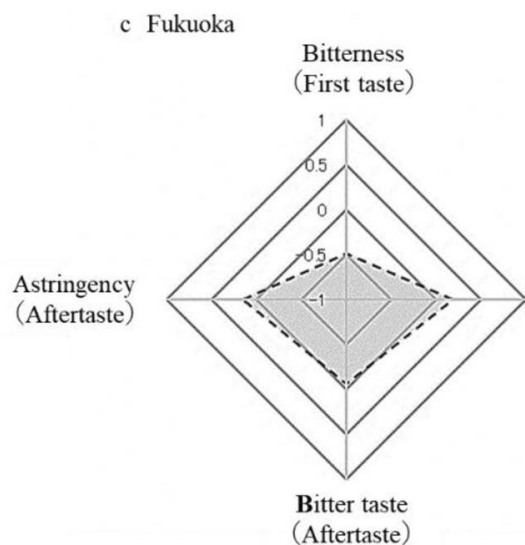

\section{b Asahi}

Bitterness

(First taste)

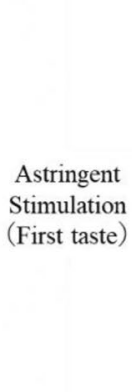

Astringent

Stimulation

(First taste)

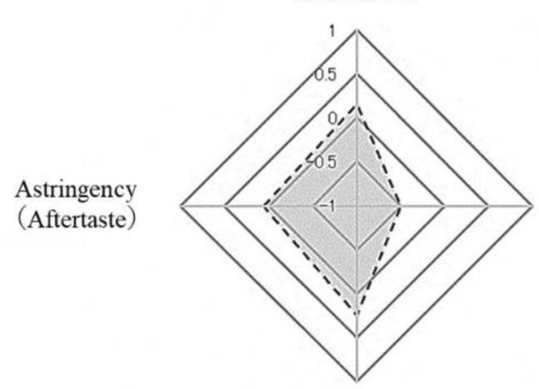

Bitter taste

(Aftertaste)
Astringent

Stimulation

(First taste)

Fig. 5 TS- $5000 Z$ analysis of bamboo shoots by production area. The results of bitter miscellaneous taste (first taste), general bitter taste (aftertaste), astringent stimulus (first taste), and astringent taste (aftertaste) of three selected samples using TS-5000Z are shown. The comparison is based on the Yuza Town sample value being set to 0.00. A greater value indicates a stronger taste

measurement and $\alpha$ ATEREE analysis (Figs. 3, 4, 5). These results suggest that there is a difference in "egumi" taste depending on the origin of bamboo shoots, not only quantitatively by chemical analysis, but also qualitatively by taste sensor. The results of chemical analysis and taste analysis showed a similar trend, suggesting that bamboo shoots from Yamagata Prefecture, which is near the northern limit of cultivation, have a stronger "egumi" taste than those from Kyoto and Fukuoka Prefectures. It is very interesting that the differences in "egumi" taste among production areas, which were empirically felt by the producers, were also shown by qualitative methods. On the other hand, analysis of astringent stimulus (first taste) and astringent (aftertaste) by taste sensor TS$5000 \mathrm{Z}$ showed that the samples from Fukuoka prefecture showed the most astringent, which was different from the results of oxalic acid concentration measurement and $\alpha$ ATEREE analysis (Figs. 3, 4, 5). In this study, since the TS-5000Z does not have a sensor to directly measure the taste of "egumi" taste and it has been described as a mixture of bitterness and astringency in past reports [26, 27], the measurements of astringent stimulus (first taste) and astringent (aftertaste) were targeted for analysis. The results suggest that when TS-5000Z reacts to the taste of oxalic acid, it may be perceived not as astringent stimulus (first taste) and astringent (aftertaste), but as bitterness (first taste) and general bitterness (aftertaste). In addition, it is thought that the substances related to the astringent stimulus (first taste) and astringent (aftertaste) measured in the samples of this study are substances other than oxalic acid.

Although there was a difference in the concentration of oxalic acid between the two samples from within the prefecture (Asahi town and Yuza town), there was almost no difference in the evaluation by $\alpha$ ASTREE. This indicates that simple concentrations of related substances do not correlate with taste, and the advantage of qualitative evaluation using taste 
sensors is again demonstrated. Furthermore, in previous reports, oxalate was said to enhance the "egumi" taste of homogentisic acid [11], but the results of this study showed that homogentisic acid was hardly present, and the "egumi" taste was linked to the concentration of oxalate, suggesting that oxalate does not simply enhance the "egumi" taste of homogentisic acid. This result is another qualitative difference that was revealed by the use of taste sensors.

As for the factors that caused differences in "egumi" taste depending on the region of production, the clones of moso-bamboo shoots in Japan are almost identical [29], therefore, the differences in taste is not related strain related. In addition, because the growth periods and sizes of the material were similar among at collection, these factors should not have greatly affected the taste. However, there are two major factors that were not standardized during the collection of materials and preparation of samples in this study: growing environment and storage management during the distribution process. For the cultivation environment, factors, such as soil and illumination owing to differences in the culm density of the parent bamboo, may be considered, but there have been no reports on a correlation between the cultivation environment and the taste of bamboo shoots. If such a correlation was clarified, then it may be possible to manipulate the taste of bamboo shoots by adjusting the cultivation environment. In addition, the storage management used during the distribution process was in accordance with the current method, which assumed that the products would be sold in Yamagata Prefecture. In the future, it will be necessary to investigate differences in taste when the same storage management conditions are used inside and outside of Yamagata Prefecture. In addition, samples from outside of the prefecture were limited to two locations, Kyoto Prefecture and Fukuoka Prefecture. More analysis of samples from outside the prefecture is needed from a variety of sources.

\footnotetext{
Acknowledgements

We would like to thank all the producers who cooperated in providing specimens. We would also like to take this opportunity to thank Mr. Tomohiro Kubo and Ms. Miho Sasaki for their assistance in sample preparation and analysis. We thank Lesley Benyon, PhD, from Edanz Group (https://en-author-services. edanz.com/) for editing a draft of this manuscript.
}

\section{Authors' contributions}

YF was responsible for all experiments, statistical analysis, and writing; $\mathrm{HN}$ performed taste analysis and contributed significantly to the writing of the manuscript; TA provided advice on chemical analysis and discussion and also wrote the manuscript. All authors have read and approved the final manuscript.

\section{Funding}

Not applicable.

\section{Availability of data and materials}

All data generated or analyzed during this study are included in this published article.

\section{Declarations}

\section{Competing interests}

The authors declare that they have no competing interests.

\section{Author details}

${ }^{1}$ The United Graduate School of Agricultural Sciences, Iwate University, 3-18 Ueda, Morioka, Iwate 020-0066, Japan. ${ }^{2}$ Yamagata Prefectural Forest Research and Instruction Center, 2707 Hei Sagae, Sagae, Yamagata 991-0041, Japan.

${ }^{3}$ Faculty of Agriculture, Yamagata University, 1-23 Wakabamachi, Tsuruoka, Yamagata 997-0037, Japan.

Received: 15 January 2021 Accepted: 18 April 2021

Published online: 27 April 2021

\section{References}

1. Shibata S (2003) Moso bamboo and Japanese (in Japanese). J Jpn Soc Reveget Tech 28(3):406-411

2. Forestry Agency (2018) Toward the promotion of bamboo utilization. https://www.rinya.maff.go.jp/j/tokuyou/take-riyou/attach/pdf/index-3. pdf. Accessed 29 Dec 2020

3. Kato K, Suzuki R, Katagiri K (2019) Demonstration of mountain village industry creation technology that shines brightly even if it is small with wild plants (in Japanese). Naganoken Ringyosogosenta Kenkyuhokoku 33:35-47

4. Ministry of Agriculture, Forestry and Fisheries (2020) Statistical survey on production of special forest products. https://www.maff.go.jp/j/tokei/ kouhyou/tokuyo_rinsan/. Accessed 16 Aug 2020

5. Yamagata Prefecture (2017) Moso bamboo cultivation management manual (Yamagata Prefecture version) (in Japanese). Yamagata Forest Research and Training Center. Sagae, Yamagata, p. 31

6. Hasegawa C, Sakamoto Y, Ichihara K (1959) On the relationship between homogentisic acid and the egumi-taste of bamboo shoots and hange. Proc Jpn Acad 35(5):246-251

7. Kozukue E (1976) Physiological and chemical studies of bamboo shoots (Part I): the changes of organic acid contents in the parts of stem, center and apical sides of bamboo shoots (in Japanese). Beacon 12:23-26

8. Kozukue E, Tsuchida H, Mizuno S (1988) Determination of homogentisic acid in bamboo shoots by column chromatography on Sephadex G-10 and reversed-phase high-performance liquid chromatography (in Japanese). J Jpn Soc Hort Sci 57(3):549-554

9. Kozukue E, Mizuno S (1989) Effects of the harvest time, size, cultivation place and storage on changes of homogentisic acid content in bamboo shoots (in Japanese). J Jpn Soc Hort Sci 58(3):719-722

10. Thammawong M, Nei D, Roy P, Nakamura N, Shiina T, Inoue Y, Hamachi H, Nonaka S (2009) Characteristics of sugar content in different sections and harvest maturity of bamboo shoots. HortScience 44(7):1941-1946

11. Kuchiba N, Sakamoto H (1990) Bamboo shoot dishes and Kyoto (in Japanese). Tyori Kagaku 23(3):263-266

12. Anjiki N (2013) Quality evaluation of Kampo prescriptions and crude drugs using a taste recognition device (in Japanese). Tokusansyubyou 16:77-83

13. Fukui $H$, Ishida T, Nishimura T, Matsuda $H$ (2006) Correlation between the results of a sensory test and an instrumental analysis of the effect of mirin for suppressing saltiness and sourness (in Japanese). Nihon Tyori Kagakkaishi 39(1):49-56

14. Ikezaki $H$ (2013) Creating a taste measure and visualizing the taste with a taste sensor (in Japanese). JVRSJ 18:93-97

15. Fujiwara T, Kokean Y (2009) Evaluation of quality degradation of pickles using taste sensors (in Japanese). Mieken Kogyokenkyusyo Kenkyu Hokoku 34:129-135

16. Toida J (2012) Evaluation of miso and soy sauces using a taste sensor (in Japanese). Nihon Jyozokyoukaishi 107(7):485-490

17. Takahashi K, Shinokura Y, Yamada K (2017) Classifcation of the Yuzukosyou using the taste sensor and examination of the flavor change by preservation (in Japanese). Miyazakiken Syokuhinkaihatusenta Kenkyuhokoku 60:37-41 
18. Toyota K, Ikezaki H, Hirabayashi K, Mimura A, Nasu K, Totsuka A (2016) Aftertaste evaluation of sake using a taste sensor (in Japanese). Nihon Jyozokyoukaishi 111(1):49-58

19. Masuno K, Shiroishi M, Nakamura M, Furukawa H (2020) Development of mushroom cultivation technology focusing on "Tastiness": quantification of the taste of Nameko mushrooms (in Japanese). Naganoken Ringyosogosenta Kenkyuhokoku 34:81-94

20. Kimura S (1963) Freeze-drying of foods (in Japanese). Sinku 6(12):470-485

21. Katayama K, Hayashi Y (1971) Research on vacuum freeze drying (in Japanese). Nihon Kikaigakkai Ronbunsyu 37(299):1387-1395

22. Yamaguchi A, Nishi R, Hirose J, Urabe K, Nadamoto T (2012) Changes in food items because of processing with different drying methods (in Japanese). Nihon Syokuhinhozou Kagakukaishi 38(3):169-176

23. Electronic Taste System ASTREE (in Japanese). https://www.alpha-mos.co. jp/sensory/am-astree-01.html. Accessed 20 Sept 2020

24. Takagi A, Kubo R, Jibiki A, Aomori T, Suzuki S, Nakamura T (2017) Identification of foods masking clindamycin bitterness using an electronic gustatory screening system followed by organoleptic examination. Iryoyakugaku 43(9):492-501
25. Taste recognition equipment (in Japanese). http://www.insent.co.jp/ products/TS5000Z_web_J.pdf. Accessed 5 Mar 2021

26. Ishii K (1966) About the bitter taste of Hatcho miso (in Japanese). Nihon Zyozo Kyokai Zasshi 61(4):314-317

27. Fujii S, Shiomi K, Furuta T, Nagai Y, Kawai T, Hirata A (2019) Taste evaluation of Kokuto (non-centrifugal cane sugar) using taste sensing system. Nihon Syokuhinkagaku Kagakukaishi 66(7):249-260

28. Maruyama E, Moteki I, Asano Y, Sueyasu K, Mineyuki K, Hashimoto K, Hasegawa C (1979) Homogentisic acid formation by p-hydroxyphenylpyruvate hydroxylase in bamboo shoot (in Japanese). Kaseigakuzasshi 30(7):603-607

29. Isagi Y, Oda T, Fukushima K, Lian C, Yokogawa M, Kaneko S (2016) Predominance of a single clone of the most widely distributed bamboo species Phyllostachys edulis in East Asia. J Plant Res 129:21-27

\section{Publisher's Note}

Springer Nature remains neutral with regard to jurisdictional claims in published maps and institutional affiliations.

\section{Submit your manuscript to a SpringerOpen ${ }^{\circ}$ journal and benefit from:}

- Convenient online submission

- Rigorous peer review

- Open access: articles freely available online

- High visibility within the field

- Retaining the copyright to your article

Submit your next manuscript at $\boldsymbol{\nabla}$ springeropen.com 